\title{
Model of strong stationary vortex turbulence in space plasmas
}

\author{
G. D. Aburjania ${ }^{1,2}$, Kh. Z. Chargazia ${ }^{1,2}$, L. M. Zelenyi ${ }^{3}$, and G. Zimbardo ${ }^{4}$ \\ ${ }^{1}$ I. Javakhishvili Tbilisi State University,2 University str., 0143 Tbilisi, Georgia \\ ${ }^{2}$ M. Nodia Institute of Geophysics, 1 Aleqsidze str., 0193 Tbilisi, Georgia \\ ${ }^{3}$ Space Research institute, Profsoyuznaya 84/32, 11710 Moscow, Russia \\ ${ }^{4}$ Physics Departament, University of Calabria, Ponte P. Bucci, Cubo 31C, 87036 Rende, Italy
}

Received: 8 January 2008 - Revised: 8 December 2008 - Accepted: 8 December 2008 - Published: 22 January 2009

\begin{abstract}
This paper investigates the macroscopic consequences of nonlinear solitary vortex structures in magnetized space plasmas by developing theoretical model of plasma turbulence. Strongly localized vortex patterns contain trapped particles and, propagating in a medium, excite substantial density fluctuations and thus, intensify the energy, heat and mass transport processes, i.e., such vortices can form strong vortex turbulence. Turbulence is represented as an ensemble of strongly localized (and therefore weakly interacting) vortices. Vortices with various amplitudes are randomly distributed in space (due to collisions). For their description, a statistical approach is applied. It is supposed that a stationary turbulent state is formed by balancing competing effects: spontaneous development of vortices due to nonlinear twisting of the perturbations' fronts, cascading of perturbations into short scales (direct spectral cascade) and collisional or collisionless damping of the perturbations in the short-wave domain. In the inertial range, direct spectral cascade occurs through merging structures via collisions. It is shown that in the magneto-active plasmas, strong turbulence is generally anisotropic Turbulent modes mainly develop in the direction perpendicular to the local magnetic field. It is found that it is the compressibility of the local medium which primarily determines the character of the turbulent spectra: the strong vortex turbulence forms a power spectrum in wave number space. For example, a new spectrum of turbulent fluctuations in $k^{-8 / 3}$ is derived which agrees with available experimental data. Within the framework of the developed model particle diffusion processes are also investigated. It is found that the interaction of structures with each other and particles causes anomalous diffusion in the medium. The effective coefficient of diffusion has a square root dependence on the stationary level of noise.
\end{abstract}

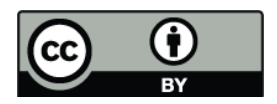

Correspondence to: G. D. Aburjania (aburj@mymail.ge)

\section{Introduction}

In a dispersive medium, especially in space, astrophysical and laboratory plasmas, the various localized wave structures could often generated and developed to the nonlinear stage (Horton, 1990; Chmyrev et al., 1991; Petviashvili and Pokhotelov, 1992; Tu and Marsch, 1997; Sudkvist et al., 2005; Alexandrova et al., 2006; Aburjania, 2006). Investigation of nonlinear interaction of wave structures with each other and with the medium is very important. Nonlinear interaction of wave structures can be described as interaction of localized structures or as a separate wave harmonics. In certain conditions, this interaction leads to the chaotization of phases both of the structures and waves. As a result of chaotic dynamics of the wave structures, the macroscopic motions usually occur in a turbulent regime.

In a real non-uniform magnetized plasma medium, the presence of spatial gradients (equilibrium density, temperature, etc.) transverse to the magnetic field leads to diamagnetic drift currents (Horton, 1990). The collective motion of particles arising due to the diamagnetic currents in such systems stimulates the development of low-frequency drift instabilities. Actually, results of the experimental observations of the spectra of low-frequency fluctuations in the magnetosphere (Sahraoui et al., 2003, 2004, 2006; Mangeney et al., 2006; Alexandrova et al.,, 2006; Narita et al., 2007), ionosphere (Lysak, 1990; Chaston et al., 1999; Stasiewicz et al., 2000; Abel et al., 2006) and experimental plasma devices (Browley and Mazzucato, 1985; Brower et al., 1987; Weissen et al., 1988; Gekelman, 1999), show that the observed fluctuations with the significant amplitude are produced due to the development of drift-Alfvén instability. One of the indications of the instability evolution in magnetized plasma is the shaping of the ordered wavy structures or vortexes, whose collective activity can finally lead to the formation of the turbulent state (Horton, 1990; Tu and Marsch, 1997; Aburjania, 2006; Alexandrova et al., 2006).

Published by Copernicus Publications on behalf of the European Geosciences Union and the American Geophysical Union. 
Following its own logic of development, in the sixties of the last century, the plasma turbulence theory was based on the weak turbulence model when only the weak interaction between modes due to nonlinearity was taken into account. Within the framework of this model, it was possible to address a wide range of questions and explain a number of important nonlinear phenomena (Kadomtsev, 1967; Horton, 1985; Galeev and Sagdeev, 1996). The weak turbulence theory was developed by expansion of the basic equations for plasma with respect to a small parameter - the ratio of the fluctuations' energy to full energy of plasma.

Unfortunately, in many interesting cases, the area of applicability of the weak turbulence theory in inhomogeneous magnetized plasma is limited because of the strong coupling of modes, and thus development of a strong turbulence theory becomes necessary. In particular, anomalous diffusion of plasma is related to the strong turbulence in the magnetic field. Therefore, in the 1970s, the primary attention in plasma turbulence theory gradually shifted to the strongly nonlinear waves and strong turbulent states (Litvak, 1983; Goldman, 1984; Shapiro and Shevchenko, 1985; Petviashvili et al., 1987; Biskamp, 2003).

According to the existing understanding, in each specific situation the strong turbulence represents to some extent a mixture of interacting waves and organized nonlinear structures (vortices). Depending on the interaction between free (weakly turbulent) waves and structures, the strong turbulence can be either mainly of wave type, or structural (vortical, granular) type (Diamond and Carreras, 1987). Herewith, these structures absorb free energy of plasma more effectively than the linear waves (Dupree, 1972; Waltz, 1985; Sagdeev et al., 1986). So the strongly localized vortex structures containing the trapped particles, propagating in plasma, can trigger the strong turbulence and increase the transport of heat and plasma particles.

The canonical representation of turbulence usually assume the existence of three, interconnected regions of interaction of wave structures with each other and the medium. The first is a seed or generation region where the development of large-scale wave structures is initiated. The second is an inertial region where the perturbation energy is cascading from long to short scales. As a rule, the energy transfer process (across different scales) is nonlinear. The third region is the damping one, where the energy dissipation of small scale structures, and accordingly, heating of medium, occurs. This process can be considered as a mechanism of transition to the stationary turbulence. In the generation region, the stationary spectrum is formed by a balance of the influx of perturbation energy (for example, due to due to the excitation of some instability) and its outflux due to nonlinear cascade. along the spectrum. . In the damping region, the process occurs via the balance of energy influx due to spectral pumping and outfluxes of energy due to dissipation. But in an inertial region, balance for a given mode occurs because of nonlinear energy cascade. In this region the fluctuation spectrum depends neither on instability, nor on the damping types (Frost and Moulden, 1977; Biskamp, 2003). Experimental investigations of turbulent motions, in particular, of temporal (in frequency domain) and especially, of spatial (in wave number domain) turbulent spectra in space plasma gave up to now only limited results, although sometimes very interesting but without establishment of cause-effect coupling between them, and without the proper theoretical foundation.

In recent years, in these experimental investigations, An essential step was made recently (2000) with the launch of four "CLUSTER" satellites (Sahraoui et al., 2003), separated from each other by the distances approximately from $100 \mathrm{~km}$ to $2000 \mathrm{~km}$ or more, depending on the period of the mission. The analysis of some observations and measurements made by Cluster was published in recent papers (Sahraoui et al., 2004, 2006; Sudkvist et al., 2005; Mangeney et al., 2006; Alexandrova et al., 2006; Zimbardo, 2006; Narita, 2007). In particular, the basic features of low-frequency turbulent state formation were thoroughly investigated. for the magnetosheath, shock wave and the high latitude cusp regions The existence of a relatively small-scale (of the order of collisionless electron skin depth), ultra low frequency (ULF) Alfvéntype wave structures $(0.1-10 \mathrm{~Hz})$ was demonstrated for the first time. The power law frequency spectrum of the energy of turbulent pulsations $F(f) \sim f^{-7 / 3}$ and a new power spectrum in a wave number space $k^{-8 / 3}$ were found (Sahraoui et al., 2006; Mangeney et al., 2006; Narita et al., 2007).

The paper is devoted to the development of a theoretical model of the stationary strong vortex turbulence in space plasma, and in particular, to the self-consistent model capable to explain the experimentally observed frequency and spatial spectra of turbulent pulsations (Sahraoui et al., 2003, 2004, 2006; Mangeney et al., 2006; Alexandrova et al., 2006; Narita, 2007). The paper is organized as follows. In Sect. 2 we obtain the initial nonlinear equations describing the dynamics of the formation of the skin size solitary drift-Alfvén vortex structures in magnetized space plasma. Analytical solution of these dynamic equations is derived and basic characteristics of ULF linear and stationary nonlinear Alfvén vortex structures are revealed. Features of vortex turbulence generation for ULF electromagnetic drift-Alfvén modes are discussed in Sect. 3. Spectra of electromagnetic vortex fluctuations are found in Sects. 3.1 and 3.2. The corresponding coefficient of anomalous diffusion which depends both on the amplitude and dispersive characteristics of a considered mode is determined in Sect. 4. At last, in Sect. 5 the basic results of the work are discussed and summarised.

\section{The initial equations and dynamics of nonlinear vor- tex structure formation}

We assume that the equilibrium state of magnetospheric plasma is characterized by single charged ions and quasinedutral particle densities, which are inhomogeneous along 
the $\mathrm{x}$-axis $\left(\nabla n_{j 0} \| x, j=e, i\right)$. Temperatures of electrons $T_{e}$ and ions $T_{i}\left(\nabla T_{e}, \nabla T_{i}=0 ; T_{e} \geq T_{i}\right)$ are assumed to be homogeneous. Inhomogeneity of equilibrium density $\left(n_{0}(x)=n_{e 0}(x)=n_{i 0}(x)\right)$ is supported by external sources (for example, an external electric field, volumetric forces, etc.). An equilibrium magnetic field $B_{0}$ is considered to be homogeneous and directed along the $\mathrm{z}$-axis $\left(B_{0} \| z\right)$.

From the point of view of strong turbulence, one of the most important types of waves in magnetized space (Lysak, 1990; Chaston et al., 1999; Stasiewicz et al., 2000; Sahraoui et al., 2003, 2004, 2006; Mangeney et al., 2006; Abel et al., 2006; Alexandrova et al., 2006; Narita et al., 2007) as well as in laboratory (Browley and Mazzucato, 1985; Brower et al., 1987; Weissen et al., 1988; Gekelman, 1999) plasmas are non-potential drift-Alfvén waves. These waves have comparatively low-frequencies (in comparison with ion cyclotron frequency $\omega_{c i}=e B_{0} / m_{i} c \gg \omega$, where $-e$ is an electron charge, $m$ - ion mass, $c$ light speed, $\omega-$ frequency of perturbations). Transverse wavelengths of these perturbations $\lambda_{\perp}$ are of the order of collisionless electron skin depth size $\lambda_{s}, \lambda_{\perp}=2 \pi / k_{\perp} \sim \lambda_{s}=c / \omega_{p e}$, where $k_{\perp}$ is a transversal wave numbers of perturbations and $\omega_{p e}=\left(4 \pi e^{2} n_{0} / m_{e}\right)^{1 / 2}$ is the plasma frequency (Kadomtsev and Pogutse, 1984; Waltz, 1985; Gekelman, 1999; Chaston et al., 1999; Mangeney et al., 2006).

Let us consider the nonlinear propagation of lowfrequency small scale $\left(\lambda_{\perp} \sim \lambda_{s}=c / \omega_{p e}\right)$ Alfvén perturbations in such inhomogeneous magnetized plasma. We express the perturbed electric $E$ and magnetic $B$ fields through the electrostatic potential $\varphi$ and the z-component of the vector potential A:

$E=-\nabla \varphi \varphi-\frac{1}{c} \frac{\partial A}{\partial t} e_{z}$

$B_{\perp}=\nabla A_{z} \times e_{z}$

where $B_{\perp}$ is the transverse component of the perturbed magnetic field, $e_{z}-$ unit vector along $\mathrm{z}$-axis. Due to the presence of inhomogeneous plasma density $\partial n_{0} / \partial x \neq 0$, electrons and ions participate in diamagnetic drift with velocities $V_{D j}=e_{y}\left(c T_{j} / q_{j} B_{0}\right) \partial n_{0} / \partial x$ (where $e_{y}$ is unit vector along an axis $y, j=e, i ; q_{e}=-e, q_{i}=e, e$ - elementary charge) and Alfvén waves become coupled to a drift modes. Waves are assumed to be quasineutral. The dynamics of nonlinear electromagnetic Alfvén type structures in such plasma is described by the system of the equations presented in the paper by Aburjania (1988). In this paper, the vortex structures with finite Larmor radius of ions are studied through the evolutionary equations, which are valid also for smaller-scale perturbations $\left(\lambda_{\perp} \sim \lambda_{s}<\rho_{i}\right.$, where $\rho_{i}=\left(T_{i} / m_{i} \omega_{c i}^{2}\right)^{1 / 2}$ is the Larmor radius of ions). Below we will be interested in wave processes with characteristic scales of the order of the electron skin length. Thus, one could use the Boltzman distribution for the description of ions and the dynamic equations from the 1988 Aburjania paper, describing drift-Alfvén structures, can be reduced to the following form:

$$
\begin{aligned}
& \frac{\partial A}{\partial t}+V_{* e} \frac{\partial A}{\partial y}+c(1+\tau) \nabla_{\|} \varphi-\lambda_{s}^{2} \frac{d_{0}}{d t} \Delta_{\perp} A=0, \\
& \frac{d_{0}}{d t} \varphi+V_{* i} \frac{\partial \varphi}{\partial y}-\frac{V_{T e}^{2}}{c \tau} \lambda_{s}^{2} \nabla_{\|} \Delta_{\perp} A=0 .
\end{aligned}
$$

Here $\quad V_{* e, i}=\mp c T_{e, i} \mathfrak{x}_{n} /\left(e B_{0}\right), \quad \mathfrak{x}_{n}=\partial \ln n_{0}(x) / \partial x$, $V_{T e}=\left(T_{e} / m_{e}\right)^{1 / 2}-$ electron thermal velocity, $\tau=T_{e} / T_{i}$, $\Delta_{\perp}=\partial^{2} / \partial x^{2}+\partial^{2} / \partial y^{2}$;

$\frac{d_{0}}{d t}=\frac{\partial}{\partial t}+\frac{c}{B_{0}}[\nabla \varphi, \nabla]_{z}$,

$\nabla_{\|}=\frac{\partial}{\partial z}-\frac{1}{B_{0}}[\nabla A, \nabla]_{z}$

In order to obtain Eqs. (3) and (4), ion parallel motion was neglected and it was assumed that the parallel current $j_{z}$ is carried only by electrons, $j_{z}=-c \Delta_{\perp} A / 4 \pi$.

Equations (3) and (4) conserve the integral of energy, $E$ :

$E=\frac{1}{2} \int\left[(\nabla A)^{2}+\lambda_{s}^{2}(\Delta A)^{2}+\lambda_{s}^{-2}\left(\frac{c}{V_{T e}}\right)^{2} \tau(1+\tau) \varphi^{2}\right] d r .(7)$

Thus, the system of Eqs. (3-7) describes the nonlinear dynamics of drift-Alfvén waves having spatial scales of the order of the skin size. The system of Eqs. (3-7) will be applied as a theoretical foundation for understanding of spacecraft observations of electromagnetic ULF Alfvén type waves in the magnetosheath (Sahraoui et al., 2006; Alexandrova et al., 2006; Mangeney et al., 2006) and the bow shock regions (Narita et al., 2007).

\subsection{Spectra of linear waves}

After the linearization of Eqs. (3) and (4), we obtain the following dispersion relation:

$$
\left(\omega-\omega_{i}\right)\left[\omega\left(1+k^{2} \lambda_{s}^{2}\right)-\omega_{e}\right]=k_{\perp}^{2} \rho_{i}^{2} k_{z}^{2} V_{A}^{2}(1+\tau),
$$

Here $\omega_{e, i}=k_{y} V_{e, i}$ are ion and electron drift frequencies, $k_{\perp}=\left(k_{x}^{2}+k_{y}^{2}\right)^{1 / 2}, k_{z}$, are perpendular and parallel (with respect to the external magnetic field $B_{0}$ ) wave numbers of perturbations, $V_{A}=B_{0} / \sqrt{4 \pi n_{0} m_{i}}$ is Alfvén speed. Equation (8) describes mutual coupling of kinetic Alfvén waves with drift waves in inhomogeneous space plasma. After neglecting the drift effects $\left(\omega \gg \omega_{e, i}\right)$ Eq. (8) reduces to the dispersion relation for the kinetic Alfvén waves:

$\omega^{2}=\omega_{k}^{2} /\left(1+k_{\perp}^{2} \lambda_{s}^{2}\right)$,

where $\omega_{k}^{2}=(1+\tau)\left(k_{z} V_{A} k_{\perp} \rho_{i}\right)^{2}$. In an electrostatic limit $\left(k_{z} \rightarrow 0\right)$, the dispersion Eq. (8) describes ion and electron drift waves:

$\omega_{1}=\omega_{i}, \omega_{2}=\omega_{e} /\left(1+k_{\perp}^{2} \lambda_{s}^{2}\right)$. 
In homogeneous plasma, in the case of $k=k_{z}=0$ the solution $\omega=0$ follows from Eq. (8), which corresponds to a convective zonal flow or a zonal magnetic field.

The frequency and spatial characteristics of drift-Alfvén waves (Eqs. 8-10) correlate well with the frequencies $(0.1-10 \mathrm{~Hz})$ and the characteristic lengths $\left(k \rho_{i}>0.5-3.5\right.$, $\lambda \sim \lambda_{s}=c / \omega_{p e}<75 \mathrm{~km}$ ) of electromagnetic Alfvén type waves obtained in spacecraft observations (Sahraoui et al., 2006; Mangeney et al., 2006; Narita et al., 2007).

It should be noted that the growth of linear Alfvén perturbations is possible for relatively long wavelengths, for example, due to dissipation instability caused by an increase of the effective medium viscosity during the scattering of high-frequency waves on plasma particles (Mikhailovskii and Pokhotelov, 1975). or due to the low-frequency modulation instability caused by the beating of two external high frequency electromagnetic waves (Aburjania, 2007). Another possible mechanism could be related with the temperatureanisotropic (mirror) instability (Sahraoui et al., 2006).

\subsection{Self-organization of nonlinear solitary vortices}

In the course of development of the above mentioned instabilities at the linear stage, the amplitude of the disturbances grows exponentially with time. With an increase of amplitude, the nonlinear effects become important, causing the twisting of wave perturbation fronts. In a dispersive media like the magnetosphere and ionosphere (see expressions 810 ), the nonlinear increase of a wave twisting can be compensated by its dispersive spreading. Then, in the medium, the stationary solitary nonlinear waves-vortices, propagating without changes of its form can be created (Horton, 1990; Petviashvili and Pokhotelov, 1992; Aburjania, 2006).

Let us show the validity of this concept by an example of an analytical solution of the nonlinear dynamic Eqs. (3), (4) in the stationary regime. For this purpose, we shall substitute into these equations auto-model variables $\eta=y-u t+\alpha z$. Here $u$ is the speed of the vortex motion velocity along the $\mathrm{y}$-axis, $\alpha \sim k_{z} / k \sim l_{\perp} / l_{\|}-$characterizes the vortex inclination angle to the z-axis, where $k \sim l_{\perp}^{-1}, k_{z} \sim l_{\|}^{-1}$ the characteristic vortex "wave numbers" are found, $l_{\perp}$ is vortex characteristic transversal linear scale (transversal according to the equinoctial magnetic field $B_{0}$ direction), and $l_{\| \mid}$is vortex characteristic linear scale along the equinoctial magnetic field direction. We shall search the stationary solution of system (3), (4) in the form of $A=A(r, \eta), \varphi=\varphi(r, \eta)$. Following (Mikhailovskii et al., 1987; Aburjania, 1988), we pass to a polar system of coordinates $r, \theta$ (where $r=\left(x^{2}+\eta^{2}\right)^{1 / 2}$, $\operatorname{tg} \theta=x / \eta$ ) and we'll divide a two-dimensional plane $r, \theta$ into internal $r<r_{0}$ and external $r \geq r_{0}$ areas. For such disturbances a nonlinear system of Eqs. (3), (4) can be reduced to a Helmholtz type equation for inner $r<r_{0}$ and outer $r \geq r_{0}$ areas of the circle via vector integration method. Further, the obtained solutions are matched continuously on the boundary $r=r_{0}$. Thus, stationary regular and strongly localized solu- tion of the system (3), (4) finally will have the form:

$$
\begin{aligned}
A(r, \theta) & =\frac{V_{T e}}{c} \varphi(r, \theta) \\
& =-\alpha B_{0} r_{0}\left[\frac{v^{2}}{\gamma^{2}} \frac{J_{1}(\gamma r)}{J_{1}\left(\gamma r_{0}\right)}-\left(1+\frac{v^{2}}{\gamma^{2}}\right) \frac{r}{r_{0}}\right] \cos \theta, \\
r & <r_{0}, \\
A(r, \theta) & =\frac{V_{T e}}{c} \varphi(r, \theta)=\alpha B_{0} r_{0} \frac{K_{1}(v r)}{K_{1}\left(v r_{0}\right)} \cos \theta, r>r_{0} .
\end{aligned}
$$

Here $J_{n}, K_{n}$ are the $n$-th order Bessel and McDonald's functions respectively, $\gamma$ is an eigenvalue specified by the boundary-value problem generated by matching the first and second derivatives with respect to $r$ of solution (11), (12) at the boundary $r=r_{0}$ :

$\gamma J_{1}\left(\gamma r_{0}\right) K_{2}\left(v r_{0}\right)+v K_{1}\left(v r_{0}\right) J_{2}\left(\gamma r_{0}\right)=0$,

and $v$ is given by:

$v^{2}=\frac{V_{* i}-\alpha V_{T e}}{\alpha V_{T e} \lambda_{s}^{2}} \tau>0$.

The solution (11-14) represents non symmetric LarichevReznik type cyclone-anticyclone double vortices (Larichev and Reznik, 1976). The vortex structures have a definite speed $u=\alpha V_{T e}$ along the y-axis, unlike the vortex from the work by Larichev-Reznik (1976), where the velocity represents a free parameter. From the condition of existence of the localized solution $v^{2}>0$ (Eq. 14) it follows that $\alpha<V_{* i} / V_{T e}$, i.e. the structure is strongly elongated along an external magnetic field $B_{0}$. With the choice of parameters specified above, $A, \varphi, \Delta_{\perp} A, \Delta_{\perp} \varphi \varphi$ appear continuous. The field of nonlinear structures consists of a cyclone-anticyclone type vortex pair of equal intensity, moving with velocity $u=\alpha V_{T e}$ along the $y$-axis and its front makes an angle with an external magnetic field of the order $\alpha \ll 1$. At large distances, the potential of the vortex structure decreases following the dependence $r^{-1 / 2} \exp (-v r)$ (see the solution 12), i.e., it is strongly localized in space. Thus, for space plasma conditions, the driftAlfvén perturbations can self-localize and self-organize in the form of solitary strongly localized nonlinear vortex structures of the electron skin depth size.

Particle oscillation (rotation) velocity in the vortex structures $(11-14) V_{E}=\left(c / B_{0}^{2}\right)\left(E \times B_{0}\right)$ is comparable or exceeds the velocity $u=\alpha V_{T e}$ of its motion. It also exceeds a phase velocity of the linear waves $V_{* e, i}$. Therefore, the vortices contain a group of trapped particles moving together with the structures. Considering that the perturbed plasma density $\tilde{n}$ is related to the potential of structure according to an expression $\tilde{n} / n_{0} \approx e \varphi / T_{i}$, from a previous condition it follows $\tilde{n} / n_{0} \sim e \varphi / T_{i} \sim e c A / T_{i} V_{T e} \sim\left(k_{\perp} L_{n}\right)^{-1}$ (where $L_{n}=\left(\partial \ln n_{0} / \partial x\right)^{-1}$ - the characteristic size of medium inhomogeneity). According to Kadomtsev (1967); Horton (1985, 1990); Aburjania (2006), the collective processes satisfying this condition are strongly turbulent. So, the vortical 
structures considered by us are the consequence of strongly nonlinear, strongly turbulent processes. These properties correlate well with the results of numerical (Sutirin, 1994; Mikhailovskaia, 1986), laboratory (Nezlin and Snezhkin, 1993; Pecseli et al., 1984) and space (Chmyrev et al., 1991; Sudkvist et al., 2005; Alexandrova et al., 2006) experiments. The given experiments show that the vortex structures of moderate amplitudes at these interactions are basically steady, keep their form at their repeated collision and poorly modify their parameters. Therefore, the above analysis is valid in a time interval, during which the integrity of nonlinear structures remains.

This is so, assuming that the solution found by us is stable enough. Structures transfer the trapped particles which move on the closed stream lines. Therefore, at the interaction (collision) of vortex structures, stream line reconnection occurs and the trapped particles will pass from one vortex to another, causing increased transfer of particles and heat across an external magnetic field. Herewith, the considered vortex structures can form a strong vortex (structural) turbulence of space plasma.

\section{Model of strong structural (vortical) turbulence}

The turbulent state described by the system of dynamic Eqs. (3) and (4) consists of small amplitude modes with a wide continuum wave number spectrum (weakly turbulent part) and also the ensemble of the vortex structures considered in Sect. 2.2. Herewith, each vortex, moving with velocity $u$ gives a certain contribution to the frequency spectrum $\omega=k u$ of fluctuations of plasma density and electromagnetic fields. As the velocity of vortex depends on amplitude, the frequency spectrum of a vortex set with various amplitudes can be wider than the corresponding spectrum of small amplitude waves poorly correlating with each other. Experimental observation in the magnetosphere (Lysak, 1990; Chaston et al., 1999; Stasiewicz et al., 2000; Sahraoui et al., 2003, 2004, 2006; Mangeney et al., 2006; Zimbardo, 2006; Abel et al., 2006; Narita et al., 2007) and in various plasma devices (Browley and Mazzucato, 1985; Brower et al., 1987; Weissen et al., 1988; Gekelman, 1999) have shown that the width of fluctuation spectrum very signifancly exceeds the one predicted by the theory of weak turbulence (Horton, 1985, 1990). Therefore, it is possible to assume, that the basic state of fluctuations spectrum in magnetized plasma is given by solitary waves and vortex solitons. As for the weak turbulent part of a spectrum, its role will be assumed negligibly small and can be added up, if necessary, to the soliton part of turbulence (we will partially consider its influence manifested in a stochastization of a vortex structure's spectrum).

Because of the strong localization of vortex structures in space, they do not produce the long-range interaction and consequently they are distributed randomly, similar to the molecules of a gas. Herewith, the random position and the phase of vortex structures are caused by collisions with each other. Such considerations allow constructing the model of strong plasma turbulence in the form of an ensemble of the vortex structures, with vortices of various amplitudes randomly distributed in space and consequently to apply a statistical approach for their description.

Thus, we shall consider that the strong turbulence of plasma represents an ensemble of weakly interacting vortex structures (the basic state), each of which is characterized by an equal distribution of energy of the system between $N$ identical vortices ( $N$ is the parameter of a state). Herewith, each vortex represents a separate degree of freedom of the system. Then, a quasi stationary turbulent state can be expanded at each given moment over the basic state of the ensemble.

\subsection{Probability of main conditions}

Let us assume that the basic state of plasma turbulence corresponds to an ensemble of two-dimensional drift-Alfvén vortices of the electron skin size: each active area of the plasma medium with a size $L \times L$ is covered by $N$ randomly distributed vortices of identical amplitude. We will notice that in a real turbulent state, the different kinds of vortices are mixed up, but both numerical (Mikhailovskaia, 1986; Sutirin, 1994), laboratory experiments (Pecseli et al., 1984; Nezlin and Snezhkin, 1993), and space observations (Chmyrev et al., 1991; Sudkvist et. al., 2005; Alexandrova et al., 2006) have shown that the vortices with essentially different amplitudes pass through each other without much interaction. So, we can assume that only the number of the vortices with the same scale is essential in a plasma, and the system of the basic states is complete. At different amplitudes, the vortices also differ by width, in this case one vortex represents for another simply the quasi classic hole; therefore their merging is hardly possible, though energy transfer is admitted.

Considering that the formation of nonlinear structures in plasma is caused by a balance of competing effects - dispersion and nonlinearity, for convenience of the analysis, we will introduce the following hierarchy for the order of magnitude of wave quantities:

$$
\begin{aligned}
& \varphi \sim A \sim O(\varepsilon), \partial / \partial y \sim \partial / \partial x \sim O\left(\varepsilon^{1 / 2}\right) ; \\
& \partial / \partial t \sim \alpha \partial / \partial y \sim \partial / \partial z \sim O\left(\alpha \varepsilon^{1 / 2}\right) ; \\
& \partial / \partial t \sim u \partial / \partial y \sim u \varepsilon^{1 / 2} \sim O\left(\alpha \varepsilon^{1 / 2}\right) \rightarrow u \sim \alpha ;
\end{aligned}
$$

here $\varepsilon$ is the small parameter describing the amplitude of the structure and is not dependent on the other small parameter $\alpha \sim l_{\perp} / l_{\|}$.

In order to avoid misunderstanding, here we mention that as far as the small parameters $\varepsilon$ and $\alpha$ are dimensionless quantities, a designation $a \sim b$ in Eq. (15) and further formulas, too, means that $a$ and $b$ are quantities of comparable order of magnitude despite of their dimension. 
A turbulence model should include the source of turbulent fluctuations, the mechanism of their attenuation and an inertial interval in which there is a noise transfer across scales to the domain of values of the parameters to where the dissipation becomes essential. Here we choose a simple model: the vortices are spontaneously born in a rather long-wave area $\left(\rho_{i}>\lambda_{\perp} \sim \lambda_{s}\right)$ with characteristic time $\tau_{n} \cong v_{n}^{-1}$ and decay due to collisional dissipation or Landau dampings in time $\tau_{d} \cong v_{d}^{-1}$. The vortex birth rate $\left(v_{n}\right)$ is balanced by their destruction rate $\left(v_{d} P(N)\right)$ in a stationary condition. From here we define the probability of a state $P(N)$ :

$P(N)=v_{n} / v_{d}$.

The rate of spontaneous birth of the vortices is defined by the time of nonlinear corrugation of a wave front $v_{n} \sim \alpha \varepsilon^{3 / 2}$. But the dissipation rate is $v_{d} \cong k_{y} V_{* i} \sim \varepsilon^{1 / 2}$ for Landau damping and isn't in document $v_{d}=$ const - for collisional dissipation. Then

$$
P(N) \sim \varepsilon^{\delta},
$$

where $\delta=1$ for Landau damping and $\delta=3 / 2$ - for collisional dissipation. The relation between the small parameter $\varepsilon$ and the parameter of the state $N$ is determined in Sect. 4 (see Eq. 28).

In the inertial range (on which a transfer of the turbulent fluctuations from excitation scales to dissipation scales occurs), the energy-containing structures are merging during their collisions. In case of merging, the increase of amplitudes of the structures occurs; and, it leads to the decrease of their transversal scale (see numerical experiments in Mikhailovskaia, 1986; Sutirin, 1994, and laboratory-space experiments in Pecseli et al., 1984; Nezlin and Snezhkin, 1993; Verkhoglyadova et al., 2003; Sudkvist et al., 2005; Alexandrova et al. 2006), i.e., there takes place a transfer of fluctuations energy into short scales.

In fact, in spacecraft observations (Sahraoui et al., 2006; Alexandrova et al., 2006; Zimbardo, 2006; Narita et al., 2007), for example, the frequency spectrum of turbulent fluctuations (dependence of ensemble average magnetic energy $\left\langle\left|B_{k}\right|^{2}\right\rangle$ on perturbation frequencies $f$ ) of ULF electromagnetic waves having a form $\left\langle\left|B_{k}\right|^{2}\right\rangle \sim f^{-7 / 3}$ is found. It obviously specifies the existence of an inertial area in the medium where the structures of different scales are present and energy transfer toward smaller scales occurs. Herewith, the spatial scale of perturbation decreases as frequency increases.

\subsection{Spatial spectra of stationary turbulence}

The generation of stationary spectra occurs as the result of the balance between the energy transfer and damping effects of the turbulent noise. We shall derive the spectra of stationary structural turbulence of two-dimensional perturbations.

It is known that the strong turbulence theory is traditionally built according to the theories of Richardson (1922), Kolmogorov (1941), which are based firstly on isotropy and homogeneity of the turbulent state and secondly on a forced averaging (Kingsep et al, 1990). This theory does not include any proper solution of the initial nonlinear dynamical equations, in spite of its obvious importance. Moreover, the characteristic scales and amplitudes of the vortices, representing the structural elements of the strong turbulence, are entered in these works without investigating a question - do these initial nonlinear equations allow the existence of the structures with such scales, amplitudes and velocities? Contrary to the works (Richardson, 1922; Kolmogorov, 1941; Iroshnikov, 1963; Kraichnan, 1965), in our model the turbulence is assumed to be anisotropic and from the very beginning (see Sect. 2.2) we build a solution of the initial dynamical equations in the form of stationary strongly localized twodimensional vortices with fully definite scales, amplitudes and velocities. Further, on the basis of these vortices, as on the turbulent carriers of perturbations, the model of the strong turbulence will be built. But, as well as in the above mentioned classical papers, we also suppose that the energy density $W$ of turbulent motion is constant for the different spectral ranges - so the energy of the large scale pulsations is transferred to the small scale ones so that energy dissipation does not actually happen in this region.

\subsubsection{Anisotropy}

A very important theory of anisotropic magnetohydrodynamic (MHD) turbulence was proposed by Goldreich and Sridhar (1995). They supposed, that MHD turbulence is strongly anisotropic due to the external magnetic field so that the turbulent transport structures (in our case - the vortices) are elongated in its direction (like the vortices found by us in Sect. 2.2; see the paragraph following Eq. 14). Correspondingly, they supposed that the energy transfer time to small scales within the system $\tau_{t r}$ is of the order of the nonlinear time or eddy turnover time $\tau_{N L}$. The Goldreich-Sridhar picture, however, does not fully agree with numerical simulations (Muller et al., 2003; $\mathrm{Ng}$ et al., 2003). From these works it is clear that it is not necessary that these two times to be equal, as was supposed by Goldreich-Sridhar. The equality of the ratio $\chi=\tau_{t r} / \tau_{N L}$ to unity seems to be very restrictive and does not correspond to some of the results stemming from direct numerical simulations where $\chi$ can be smaller than unity, as observed by $\mathrm{Ng}$ et al. (2003) and Muller et al. (2003). We as well as other authors (for example, Galtier et al., 2005; Bolddyrev, 2005), suppose $\chi$ to be a constant for all scales but not necessarily equal to unity (the critical balance condition). Solar wind (Matthaeus et al., 1995) and magnetosheath data (Mangeney et al., 2006), where $\chi$ seems to be smaller than unity, support the validity of our assumptions.

As it was mentioned above, in agreement with the Kolmogorov-Iroshnikov-Kraichnan and further MHD theories (Schekochihin et al., 2007), we assume that the cascade of Alfvénic turbulence energy is local in space and the flux 
of energy through the transversal (with respect to the background magnetic field $B_{0}$ direction) scale $l_{\perp}$ in the inertial range is scale-independent:

$$
\left(\frac{B_{l_{\perp}}}{B_{0}}\right)^{2} \frac{V_{A}^{2}}{\tau_{t r}} \sim W_{B}=\text { const, }
$$

where $W_{B}$ is the unit volume energy flux, the subscript $l_{\perp}$ indicates fluctuations associated with the perpendicular scale $l_{\perp}$, and $\tau_{t r}$ is the energy transfer time for the beginning of the cascade processes. It is now assumed that the turbulence is strong, i.e. that the Alfvénic linear propagation terms are comparable to the nonlinear terms in the dynamical Eqs. (3$6)$ :

$$
\frac{\partial}{\partial z} \sim \frac{B_{l_{\perp}} \cdot \nabla}{\left|B_{0}\right|} \Rightarrow \frac{B_{l_{\perp}}}{B_{0}} \sim \frac{l_{\perp}}{l_{\|}} .
$$

This is the critical-balance condition, applied scale by scale. Assuming that the cascade time is comparable to the inverse frequency of kinetic Alfvén waves (see Eq. 9) $\tau_{t r}=\tau_{A} \sim \omega_{k}^{-1} \sim l_{\|} l_{\perp} / V_{A} \rho_{i} \sim \chi \tau_{N L}$ and introducing "the wave vector analogs" of orthogonal and transversal linear sizes of the vortices $-k_{\perp}=2 \pi / l_{\perp}, k_{\|}=2 \pi / l_{\|}$, then from Eqs. (18), (19) we'll have:

$k_{\|} \sim\left(\frac{W_{B}}{V_{A}^{3} \rho_{i}}\right)^{1 / 3} k_{\perp}^{1 / 3}$.

If we explicitly use the fact that the rates of energy transfer per unit volume $W_{B}$ do not depend on the scale, we finally obtain

$k_{\|} \sim \frac{k_{\perp}^{1 / 3}}{B_{0}^{2 / 3}}$.

This relation shows that the considered turbulence is intrinsically anisotropic. These scaling have been confirmed by numerical simulation of electron MHD (EMHD) turbulence (Cho and Lazarian, 2004; see also Schekochihin, et al., 2007). It is obvious that turbulence develops more freely in background magnetic field direction. The size of the anisotropy and the strength of the expressed orthogonal spreading of turbulence change in accordance with the strength of the magnetic field induction. So, the magnetic field induces the anisotropies of compressible MHD turbulence. Anisotropy increase with the scale decrease was predicted for Alfvénic motion by Goldreich and Sidhar (1995) and confirmed numerically for compressible MHD in Cho and Lazarian (2003). This conclusion fully confirms the Cluster observations and measurements of the turbulent processes in the solar wind, magnetosheath and foreshock regions (Sahraoui et al., 2006; Mangeney et al., 2006; Alexandrova et al., 2006; Narita et al., 2007). From Eqs. (20) and (21) it is obvious that a weakly anisotropic state, $l_{\|} \sim l_{\perp}$, forms in conditions of not very strong mean magnetic field $B_{0}$, when $V_{A}^{3} \sim W_{B} / \rho_{i}$.

\subsubsection{Compressibility}

In order to obtain the system of Eqs. (3), (4) for the description of nonlinear dynamics, we took into account that the linear scale of wave structures is less than or equal to ion Larmur radius $\left(l_{\perp} \sim \lambda_{s} \sim \rho_{i}\right)$. For such perturbations in inhomogeneous plasma, the ions are mainly distributed according to Boltsman's Law $n_{i} \sim n_{0} \exp \left(-e \varphi / T_{i}\right)$. Therefore, taking into account the condition of quasi neutrality and expressions (2), (11) and (12), we understand that the considered medium experiences density fluctuations, i.e., the medium are compressible. Consequently, the density of perturbed charged particles $\rho=m_{i} n$ can be expressed by the formula $\rho \sim m_{i} n_{0} e \varphi / T_{i} \sim m_{i} n_{0} e c A / T_{i} V_{T e}$ (see second paragraph after Eq. 14). The energy density must be used in a compressible medium to describe the abovementioned cascade of energy, i.e., energy per unit volume (as it was made in the previous item) (Fleck, 1996; Kowal and Lazarian, 2007; Alexandrova, 2008), instead of the unit mass energy in order to take into account the medium fluctuations.

In the strong turbulent medium, as we mentioned above, in the turbulent cascade, energy transfers from comparably large-scale nonlinear structures to smaller scale vortices. As the scale of vortex structures decreases, the values of nonlinear terms become significant in the dynamic Eqs. (3), (4) (see the last terms therein). Consequently, the time-scale of nonlinear interaction $\tau_{N L}$ (i.e. turnover time) can be estimated by balancing the first and last terms of Eq. (4). At the same time, if we take into account that the plasma density fluctuations $\rho_{l_{\perp}}$ are related to an electrostatic potential $\varphi_{l_{\perp}}$ via Boltzmann relation $\varphi_{l_{\perp}} \sim\left(T_{i} / e m_{i} n_{0}\right) \rho_{l_{\perp}}$, and the magnetic field perturbation $-B_{l_{\perp}} \sim A / l_{\perp}$ and taking also into account the fact that in such strongly nonlinear turbulence state $-\partial \Delta_{\perp} A / \partial z \leq[\nabla A, \nabla]_{z} \Delta A / B$, $B \sim B_{0} \sim\left[\nabla A, e_{z}\right] \sim k_{\perp} A \sim A / l_{\perp}$ (see Schekochihin, et al. 2007), from Eq. (4) we get:

$\tau_{N L} \sim l_{\perp}^{2} \frac{\rho_{l_{\perp}}}{B_{l_{\perp}}}$.

From this equality, it is clear that as the energy cascades to the small scale modes, i.e. as $l_{\perp} \rightarrow 0$, also $\tau_{N L} \rightarrow 0$. This means that in the inertial region during the development of the turbulent cascade processes, the time $\tau_{N L}$ becomes smaller in comparison with other characteristic time-scales. Thus, it is clear that, in the inertial region in the case of well developed turbulence using energy balance Eq. (18) (which is valid for ensembles of all scale structures) $\tau_{t r}$ must be replaced by $\tau_{N L}$ according to Eq. (22):

$W_{B} \sim\left(\frac{B_{l_{\perp}}}{B_{0}}\right)^{2} \frac{V_{A}^{2}}{l_{\perp}^{2}} \frac{B_{l_{\perp}}}{\rho_{l_{\perp}}} \sim \frac{B_{l_{\perp}}^{3}}{l_{\perp}^{2} \rho_{l_{\perp}}}=$ const.

In cascade-hierarchic turbulent spectra formation, in order to take into account the medium compressibility, we have to use the approach taken by Weizsacker (1951), which was concerned with hierarchic cloud stair formation (see also 
Fleck, 1996; Kowal and Lazarian, 2007). According to this approach, each large cloud consists of a certain number of smaller clouds, which contain even smaller clouds. In our problem, the strongly localized vortices are assumed to be under the clouds. So, according to this model, the mass density of the vortices $\rho$ at two successive levels of the hierarchy is related to the their corresponding scale size $l$ by the following equation

$$
\frac{\rho_{v}}{\rho_{v-1}}=\left(\frac{l_{v}}{l_{v-1}}\right)^{-3 \mu},
$$

where $\rho_{v}$ is the average density inside a vortex at level $v, l_{v}$ is the mean size of that vortex, $\mu$ is constant and a measure of the degree of compression at each level $v$ (larger $v$ meaning larger length scale), and ranges from $\mu=0$ for no compression up to $|\mu|=1$ for isotropic compression $(3|\mu|$ is the number of dimensions in which the compression takes place). Thus, using this density scaling, $\rho_{l_{\perp}} \sim\left(l_{\perp}\right)^{-3 \mu}$ from an expression to a constant spectrum energy transfer rate (Eq. 23) we have

$B_{l_{\perp}} \sim l_{\perp}^{2 / 3-\mu}$

Thus a medium compressibility $(\mu \neq 0)$ significantly influences the spatial spectra of the turbulence.

\subsubsection{Turbulent spectrum}

The relation (25) obtained above allows the determination of the energy spectra $E\left(k_{\perp}\right)$ of the strong vortex turbulence as a function of the to transversal "wave vector" $k_{\perp}$ similar to the work (Alexandrova, 2008). For this purpose let us use the formula of perturbation's energy (Eq. 7), from which for transversal cascades it follows (after averaging in longitudinal direction), that $E(k) k_{\perp} \sim B_{k_{\perp}}^{2}$. Substituting the expression for $B$ from Eq. (25) in Fourier space into the latter, we finally obtain:

$E\left(k_{\perp}\right) \sim \frac{B_{l_{\perp}}^{2}}{k_{\perp}} \sim k_{\perp}^{-7 / 3+2 \mu}$.

In the incompressible plasma limit $(\mu=0)$, this phenomenology predicts a spectrum $k^{-7 / 3}$. Such a spectrum has been observed both in direct numerical simulations of an incompressible EMHD turbulent system (Biskamp et al., 1996, 1999) and in the EMHD limit of the incompressible Hall MHD shell heuristic model (Galtier and Buchlin, 2007). In the case of isotropic compressions toward smaller scales $(\mu=1)$, which can take place in the interstellar medium, the spectrum is $E(k) \sim k^{-1 / 3}$. If the isotropic compression is going on toward large scales $(\mu=-1)$, the spectrum will be $E(k) \sim k^{-13 / 3}$, which was confirmed by the numerical calculations for the conditions of the solar wind (Galtier and Buchlin, 2007) (see also Alexandrova, 2008). Recently, new energy spectra of turbulence $E(k) \sim k^{-8 / 3}$ were found by the Cluster mission in the magnetosheath (Sahraoui et al., 2003,
2004, 2006), in the foreshock-region (Narita et al., 2007), and in the solar wind (Howes et al., 2008) correspond to a value of plasma compressibility degree $\mu=-1 / 6$. Generally, the value of $\mu$ for a certain medium has to be determined by appropriate observations and measurements or on the basis of corresponding exact numerical modelling.

\section{Anomalous diffusion of plasma particles due to vortices}

In experimental magnetically confined plasma devices, anomalously large thermal conductivity and diffusion of particles are observed (Liewer, 1985; Huld et al., 1988). This effect is caused by the development of strong turbulence. Herewith, the obtained values of the turbulent diffusion coefficient lay between the values of the classical diffusion coefficient $D_{c}=\rho_{e}^{2} / \tau_{e i} \sim n B_{0}^{-2} T_{e}^{-1 / 2}$ (where $\rho_{e}$ is the electron Larmor radius, $\tau_{e i}$ - time of electron scattering on ions) and of the Bohm diffusion coefficient $D_{B}=c T_{e} /\left(16 e B_{0}\right)$. The reason for such an anomalously large transfer (in comparison with the classic one) in plasma can be the regular vortex structures considered above. Really the vortex structures contain the trapped particles and, diffusing in plasma, interacting with each other and the plasma particles can lead to a rather strong increase in transversal diffusion and thermal conductivity of plasma. The vortex diffusion coefficient can be estimated by the following expression $D_{v}=l_{\perp}^{2} / \tau_{v}$ (where $l_{\perp}$ is the transversal scale of a vortex, $\tau_{v}$ - characteristic time of interaction of the vortices). Thus it is quite probable, that $D_{v} \gg D_{c}$. Let us consider this question in more detail.

Let $W$ be the average density of perturbation energy. In a state of stationary turbulence there is a constant stream of energy from large-scale fluctuations to small-scale ones, and, in general, the average energy density $W$, is not a constant along the spectral range. However, if the characteristic time of energy density variation $t_{0}$ is large enough, $t_{0} \gg\left(k_{y} \alpha V_{T e}\right)^{-1}$, then, as follows from the dynamic Eqs. (3), (4), it is possible to assume that in the time intervals interesting for us $W=$ const, and to assume that the perturbation energy is equally distributed over all degrees of freedom (on the existing structures). So designating the energy of each vortex through $E_{v}$ (see Eq. 7), we have:

$$
\begin{aligned}
& W L^{2}=N E_{v}=N \varepsilon^{2} I, \\
& E_{v}=\frac{1}{2} \int\left[\left(\nabla A_{v}\right)^{2}+\lambda_{s}^{2}\left(\Delta A_{v}\right)^{2}+\tau(1+\tau) \frac{\omega_{p e}^{2}}{V_{T e}^{2}} \varphi_{v}^{2}\right] . \\
& d x d \eta=\varepsilon^{2} I .
\end{aligned}
$$

Here $A_{v}(x, \eta), \varphi_{v}(x, \eta)$ are the stationary solutions of the form (11-12); $I-$ a constant quantity of the order of one. From the relation (27) we get the expression for the $\varepsilon$ small parameter:

$\varepsilon \sim W^{1 / 2} L N^{-1 / 2}$. 
The characteristic scale of the vortex structures is given by

$l \sim \lambda_{s} \sim \varepsilon^{-1 / 2} \sim W^{-1 / 4} L^{-1 / 2} N^{1 / 4}$.

The maximum number of the vortices is defined by the condition of dense packing

$N_{\max } \sim L^{2} / l^{2} \sim W^{1 / 3} L^{2}$.

The minimum number is found from a condition $\varepsilon\left(N_{\min }\right)<1$. So,

$N_{\min } \sim W L^{2}$.

Thus, by means of Eqs. (27), (28), (29), we have estimates for a characteristic number of vortices $N_{0}$, effective wave number $k_{0}$ and amplitude $\varphi_{0}$ of the structures:

$k_{0} \sim N^{1 / 2}, \quad \varphi_{0} \sim N \sim k_{0}^{2}, \quad N_{0} \sim W L^{2} k_{0}^{-4}$.

Knowing the energy density of the vortices $W$, it is possible to study the influence of vortex structures on the processes of heat and plasma transport in magnetized plasmas. We shall define the diffusion coefficient of the test particles of plasma at their interaction with an ensemble of vortex structures. With this purpose we shall introduce the characteristic temporal scales of the processes causing diffusion of the test particles: the frequency of collision between test particles and $N$ vortices $\left(v_{c}\right)$, the frequency of pair collisions between the vortices $\left(v_{v}\right)$, and the time of interaction between particles and a vortex $\left(\tau_{i} \sim l_{\perp} / u\right)$. For a definition of collision frequencies of the vortices (quasi particles) with each other and test particles, the corresponding expression for the classical pair collisions was used, $v z=z \sigma n_{v} v_{\text {rel }}$ (where $\sigma$ is the section of scattering, $n_{v}$ - density of quasi particles, $v_{\text {rel }}-$ speed of relative motion, $v_{\text {rel }} \sim u$ ). Taking into account that the vortex structures are two-dimensional, we define the vortex gas density as $n_{v}=N / L^{2}$; assuming the section of scattering $\sigma$ to be of the order of the vortex scale $\left(\sigma \sim l_{\perp}\right)$ (Horton, 1985, 1990), we find an expression for $v_{v}$ and $v_{c}$

$v_{c}(N)=\sigma n_{v} u \sim \alpha W^{-1 / 4} L^{-5 / 2} N^{5 / 4}$,

$$
\begin{aligned}
v_{v}(N)= & \int_{N_{\min }}^{N_{\max }}\left|V(N)-V\left(N_{1}\right)\right| \sigma\left(N_{1}\right) n_{v}\left(N_{1}\right) P\left(N_{1}\right) d N_{1} \\
& \sim \alpha W^{2 / 3} L N^{-1 / 2} .
\end{aligned}
$$

Here $V$ is the vortex structure velocity, equal to $V \sim u(1+\varepsilon(N))$. This value of the velocity is defined from the formulas (14) and (15) appropriate for the vortex (Eq. 12) characteristic scale.

The nonlinear interaction between the vortices leads to decorrelation of the particles and the field, therefore diffusion of the test particles is not purely quasi linear. The test particles decouple from the vortices during the effective correlation time $\tau_{c}$ :

$\tau_{c} \sim v_{c}^{-1} /\left(v_{v} \tau_{i}\right) \sim \alpha^{-1} W^{-1 / 6} L^{2} N^{-1}$.
The correlation length is approximated by the transversal scale of a vortex $l_{c} \sim l_{\perp}$. By means of the Eq. (35) we can define the contribution of a single vortex to the diffusion coefficient:

$D_{N}=\frac{l_{c}^{2}}{\tau_{c}} \sim \alpha W^{-1 / 3} L^{-3} N^{3 / 2}$.

Knowing the expression (17) for the probability $\mathrm{P}(\mathrm{N})$, the total diffusion coefficient can be obtained simply as:

$D_{v}=\int_{N_{\min }}^{N_{\max }} D_{N}(N) P(N) d N \cong \alpha W^{1 / 6}$.

The value of the diffusion coefficient (37) does not depend on the dissipation form and has an identical order for all $0<\delta<2$. For conditions of a moderate noise level $W$, diffusion of plasma particles becomes anomalous and the diffusion coefficient has the same order, as for the nonlinear convective cells (Weiland, 1977). We point out that according to Eq. (32) the expressions $W \cong N \varphi_{0}^{2} \sim \varphi_{0}^{3}$ and $D_{v} \sim \alpha \sqrt{\varphi_{0}}$ should be valid. The specificity of a vortex in the diffusion coefficient is attributed to the parameter $\alpha$ related with the displacement speed of structure $u, \alpha=u / V_{T e}$ (i.e. the initial condition of a structure formation or a primary diffusion coefficient), and also the size $\varphi_{0}$ defining rotation velocity of the trapped particles in a vortex $V_{0}=k c \varphi_{0} / B_{0}$. The value obtained for the effective diffusion coefficient of the structural turbulence (i.e. having the root dependence on the fluctuation amplitude) will be well correlated with the results of earlier theoretical works (Isichenko et al., 1989; Rosenbluth et al., 1987) (though these works also considered other models) and experimental observations (Liewer, 1985; Huld et al., 1988).

It's interesting to compare this analysis with the existing results and observations in laboratory, interplanetary space, magnetosphere and ionosphere and to reveal peculiar features of the particle and heat transport, in particular to determine the real values of the diffusion coefficient and to compare them to theoretical values of an effective coefficient of anomalous vortex diffusion (Eq. 37). Therefore, there is an obvious strong motivation to continue such observations and measurements in the future.

\section{Discussion of results}

In this work, we investigated collective processes in magnetized plasma, caused by nonlinear regular structures. It is shown that in space plasmas, relatively short wavelength (with length of the order of collision less skin depth) drift Alfvén type waves could be excited and self-localized into nonlinear spatially two-dimensional vortex structures. The vortices are significantly elongated in the direction of the mean magnetic field $\left(l_{\|} \gg l_{\perp}\right)$ which well correlates with satellite observation data. 
These electromagnetic small-scale vortex structures, carrying trapped particles and spreading in plasma, generate the strong turbulence having granular character. Turbulence is represented by a gaseous ensemble of $N$ strongly localized, weakly interacting identical vortices forming the background state. Turbulence excites appreciable fluctuations of density, velocity, magnetic and electric fields and intensifies the transfer processes. Thus, the width of a strong vortical turbulence spectrum is much larger than the value predicted by the weak turbulence theory. This theory is in good agreement with experimental observations.

It is shown that the turbulent processes mentioned in this paper are characterized by strong anisotropy and this factor is caused by the influence of the local mean magnetic field. Particularly, in the magnetosheath for coupling between transversal $k_{\perp}=2 \pi / l_{\perp}$ and longitudinal $k_{||}=2 \pi / l_{\|}-$ the components of the characteristic "wave vectors" of the vortex turbulence with respect to the local mean magnetic field - the following relation is obtained: $k_{\|} \sim k_{\perp}^{1 / 3} / B_{0}^{2 / 3}$. Because of this, the turbulence develops more effectively in transverse direction to the local magnetic field. This anisotropy is essentially as strong as this magnetic field. This theoretical result is also well correlated with satellite measurements in the magnetosphere and results of appropriate numerical calculations.

It is established that energy spectra of the strong turbulence in the wave-vector space has a power law form $E\left(k_{\perp}\right) \sim k_{\perp}^{-7 / 3+2 \mu}$, where the parameter $\mu=$ const, $l \geq|\mu| \geq 0$ and characterizes the degree of local compressibility. This formula clearly describes the turbulent spectrum behaviour found in numerous experiments and numerical calculations. Namely, for a recently found turbulent spectrum $E\left(k_{\perp}\right) \sim k_{\perp}^{-8 / 3}$ from Cluster satellites, the corresponding compressibility degree in the magnetosheath must be $\mu=-1 / 6$.

As already noted in Sect. 3.2, it must be mentioned that in recent theoretical works, different approaches to strong turbulence were proposed. In electron magnetic hydrodynamics (EMHD) and Hall MHD, on the basis of numerical solutions and heuristic analysis of a two and three-dimensional system of dynamical equations in the papers by (Biskamp et al., 1996, 1999; Cho and Lazarian, 2003, 2004), the energetic spectra of the turbulent pulsations $k^{-5 / 3}$ and $k^{-7 / 3}$ were obtained for a different range of wave numbers. In the papers by Galtier et al. (2007); Alexandrova (2008); Howes et al. (2008) where numerical solutions of the system of equations for the dynamics of solar wind and magnetosheath were performed, analogous power spectra $k^{-5 / 3}, k^{-7 / 3}$ and $k^{-11 / 3}$ for magnetic fluctuations in the different range of the wave numbers were obtained. In Galtier et al. (2005) and Boldyrev (2005), with the help of phenomenology analysis, it was established that in magneto hydrodynamics the existence of strongly anisotropic spectra is allowed, which essentially depends on the horizontal $k_{\perp}\left(\right.$ where $k_{\perp}=\left(k_{x}^{2}+k_{y}^{2}\right)^{1 / 2}$ ) as well as on the vertical $k_{z}$ components of the wave vector: for non-dimensional energy $E\left(k_{\perp}, k_{z}\right)$ a correlation was found, $E\left(k_{\perp}, k_{z}\right) \sim k_{\perp}^{-m} k_{z}^{-n}$, where $3 m+2 n=7$. It is highly desirable to continue this theory and data comparison in future experiments and in more complete numerical models.

It is demonstrated theoretically that strong turbulence in the plasma media creates significantly strong fluctuations of density, electric and magnetic fields. At the same time, the generated collective activity causes a pronounced enhancement of energy, heat and mass transfer processes. Mutual interaction of the nonlinear vortex structures and medium particles causes anomalous diffusion (in comparison with classical collisional transport) in plasma. The effective coefficient of the structural turbulent diffusion $D_{v}$ has a square root dependence on the characteristic amplitude of the stationary noise $\varphi_{0}, D_{v} \sim \sqrt{\varphi_{0}}$, and essentially differs from the results of the quasi linear theories, in which $D \sim \varphi_{0}^{2}$.

Further observations and measurements of existing diffusion transfer processes in space plasma are required for the determination of the real diffusion coefficient and validation of their agreement with theoretically obtained values. The analysis of strong vortex turbulence in this work is in agreement with known results of numerical, space and laboratory experiments. This supports our conjecture that the strong structural turbulence is responsible for the formation of frequency and spatial spectra of electromagnetic fields and controls the anomalous transfer of particles and heat observed in magnetized plasma media.

Acknowledgements. This research was supported in part by the INTAS grant 06-1000017-8934, Russian program for scientific schools RF NS 4722008.2 and by the Italian Space Agency, contract ASI n. I/015/07/0 "Esplorazione del Sistema Solare", and by the Italian INAF.

Edited by: V. Shrira

Reviewed by: two anonymous referees

\section{References}

Abel, G. A., Freeman, M. P., and Chisham, G.: Spatial structure of ionospheric convection velocities in regions of open and closed magnetic field topology, Geophys. Res. Lett., 33, L24103, doi:10.1029/2006GL027919, 2006.

Aburjania, G. D., Mikhailovskii, A. B., and Lakhin, V. P.: Nonlinear Regular Structures of Drift Magnetoacoustic Waves, Jo. Plasma Phys., 38, 373-386, 1987.

Aburjania, G. D.: Electromagnetic drift vortices in a rotating plasma cylinder, Physica Scripta, 38, 59-63, 1988.

Aburjania, G. D.: Structural turbulence and diffusion of the plasma in magnetic trap, Plasma Phys. Rep., 16., 70-76, 1990.

Aburjania, G. D.: Self-Organization of the Nonlinear Vortex Structures and the Vortical Turbulence in the Dispersive Media: KomKniga, Editorial URSS, Moscow, 2006.

Aburjania, G. D.: Nonlinear generation mechanism for the vertical electric field in magnetized plasma media, Phys. Plasmas, 14, 1-7, 2007. 
Alexandrova, O., Mangeney, A., Maksimovich, M., CornilleauWehrlin, N., Bosqued, J.-M., and Andre, M.: Alfvén vortex filaments observed in magnetosheath downstream of a quasiperpendicular bow shock, J. Geophys. Res., 111, A12208, doi:10.1029/2006JA011934, 2006.

Alexandrova, O.: Solar wind vs magnetosheath turbulence and Alfvn vortices, Nonlin. Processes Geophys., 15, 95-108, 2008, http://www.nonlin-processes-geophys.net/15/95/2008/.

Biskamp, D., Schwarz, E., and Drake, J. F.: Two-dimensional electron magnetohydrodynamic turbulence, Phys. Rev. Lett., 76, 1264-1267, 1996.

Biskamp, D., Schwarz, E., Zeiler, A., Celani, A., and Drake, J. F.: Electron magnetohydrodynamic turbulence, Phys. Plasmas., 6, 751-758, 1999.

Biskamp, D.: Magnetohydrodynamic Turbulence, Cambridge University Press, 2003.

Boldyrev, S.: On the spectrum of magnetohydrodynamic turbulence, The Astrophys. J., 626, L37-L40, 2005.

Browley, T. and Mazzucato, E.: Scaling of density fluctuations PDX, Nucl. Fusion, 25, 507-524, 1985.

Brower, D.L., Peebles, W. A., and Luhmann, N. C.: The spectrum, spatial distribution and scaling of microturbulence in the Texas Tokamak, Nucl. Fusion, 27, 2055-2073,1987.

Chaston, C. C., Carlson, C. W., Ergun, R. E., and McFadden, J. P.: FAST observations of inertial Alfvén waves in the dayside aurora, Geophys. Res. Lett., 26, 647-650, 1999.

Chmyrev, V. M., Marchenko, V. A., Pokhotelov, O. A., Stenflo, L., Streltsov, A. V., and Steen, A.: Vortex structures in the ionosphere and the magnetosphere of the Earth, Planet. Space Sci. 39, 1025-1037, 1991.

Cho, J. and Lazarian, A.: Compresible magnetohydrodynamic Turbulence: mode coupling, scaling relations, anisotropy, viscositydamped regime, and astrophysical implication, Mon. Not. Astron. Soc., 345, 325-341, 2003.

Cho, J. and Lazarian, A.: The anisotropy of magnetohydrodynamic turbulence, The Astrophys. J., 615, L41-L44, 2004.

Diamond, P. H. and Carreras, B. A.: On mixing length theory and saturated turbulence, Comm. on Plasma Phys. Contr. Fus., 10, 271-278, 1987.

Dupree, T. H.: Theory of phase space density granulation on plasma, Phys. Fluids, 15, 334-344, 1972.

Fleck Jr., R. C.: Scaling relations for the turbulent, non-selfgravitating, neutral component of the interstellar medium, The Astrophys. J., 458, 739-741, 1996.

Frost, W. and Moulden, T. H. (Eds.): Handbook of Turbulence, Plenum Press, New York and London, 1977.

Galeev, A. A. and Sagdeev, R. Z.: Nonlinear Theory of plasma, in: Reviews of Plasma Physics, 7, edited by: Leontovich, M. A., Consultant Bureau, New York, 1976.

Galtier, S., Pouquet, A., and Mangeney, A.: On spectral scaling laws for incompressible anisotropic magnetohydrodynamic turbulence, Phys. Plasmas, 12, 092310, doi:10.1063/1.2052507, 2005.

Galtier, S. and Buchlin, E.: Multiscale Hall-magnetohydrodynamic turbulence in the solar wind, The Astrophys. J., 656, 560-566, 2007.

Gekelman, W.: Review of laboratory experiments on Alfvén waves and their relationship to space observations, J. Geophys. Res, 104, 14417-14435, 1999.
Goldman, M. V.: Strong turbulence of plasma waves, Rev. Mod. Phys., 56, 709-735, 1984.

Goldreich, P. and Sridhar S.: Toward a theory of interstellar turbulence. II. Strong Alfvénic turbulence, The Astrophys. J., 438, 763-775, 1995.

Gruzinov, A. B. and Pogutse, O. P.: Description of the plasma turbulence in the strong magnetic field, Dokl. ASSR, 290, 322-325, 1986.

Horton, W.: Drift Turbulence and anomalous transport, in: Basic Plasma Physics, 2, edited by: Galeev, A. A. and Sudan, R. N., North-Holand, Amsterdam, 1985.

Horton, W.: Nonlinear Drift Waves and Transport in Magnetized Plasma: Institute for Fusion Studies the University of Texas at Austin, IFSR, Review, Austin, Texas, 1990.

Howes, G. G., Cowley, S. C., Dorland, W., Hammett, G. W., Quataert, E., and Schekochihin, A. A.: A model of turbulence in magnetized plasmas: Implications for the dissipation range in the solar wind, J. Geophys. Res., 113, A05103, doi:10.1029/2007.JA012665, 2008.

Huld, T., Lizuka, S., Pecseli, H. L., and Rasmussen, J. J.: Experimental investigation of flute-type electrostatic turbulence, Plasma Phys. Contr. Fusion, 30, 1297-1318, 1988.

Isichenko, M. B., Kalda, Y. L., Tatarinova, E. B., Telkovskaia, E. B., and Yankov, B. B.: Diffusion in a medium with the vortex motion, JETP, 96, 913-925, 1989.

Iroshnikov, R. S.: The turbulence of a conducting fluid in a strong magnetic field, Astr. Zh., 40, 742-750, 1963 (English transl. Soviet Astron., 7, 566-574, 1964).

Kadomtsev, B. B.: Plasma turbulence, in: Reviews of Plasma Physics, 4, edited by: Leontovich, M. A., Consultant Bureau, New York, 1967.

Kadomtsev, B. B. and Pogutse, O. P.: Theory of the electron transfer processes in strongly magnetized field, Pisma v JETP, 39, 225228, 1984.

Kingsep, A. S., Chukbar, K. V., and Yan'kov, V. V.: Electron magnetohydro-dynamics, in: Reviev of Plasma Physics, 16, edited by: Kadomtsev, B. B., Consultant Bureau, New York, 1990.

Kolmogorov, A. N.: Local structure of turbulence in the noncompresible viscous fluid at very high Reynolds number, Dokl. Akad. Nauk. SSSR, 30, 299-303, 1941.

Kowal, G. and Lazarian, A.: Scaling relations of compressible MHD turbulence, The Astrophys. J. Lett., 666, L69-L72, 2007.

Kraichnan, R. H.: Inertial range spectrum hydromagnetic turbulence, Phys. Fluids, 8, 1385-1387, 1965.

Larichev, V. D. and Reznik, G. M.: On the two dimensional solitary Rossby Waves, Dokl. ASSR., 231, 1077-1079, 1976.

Liewer, P. C.: Measurement of microturbulence in tokamaks and comparison with theories of turbulence and anomalous transport, Nucl. Fusion, 25, 543-621, 1985.

Litvak, A. G.: Dynamic nonlinear electromagnetic phenomena in the plasma, in: Reviews of Plasma Physics, 10, edited by: Leontovich, M. A., Consultant Bureau, New York, 1983.

Lysak, R. L.: Electromagnetic coupling of the magnetosphere and ionosphere, Space Sci. Rev., 52, 33-87, 1990.

Mangeney, A., Lacombe, C., Maksimovic, M., Samsonov, A. A., Cornilleau-Wehrlin, N., Harvey, C. C., Bosqued, J.-M., and Trávnícek, P.: Cluster observations in the magnetosheath - Part 1: Anisotropies of the wave vector distribution of the turbulence 
at electron scales, Ann. Geophys., 24, 3507-3521, 2006, http://www.ann-geophys.net/24/3507/2006/.

Matthaeus, W. H., Oughton, S., Pontius, D. H., and Zhou, Y.: Evolution of energy-containing turbulent eddies in the solar wind, J. Geophys. Res., 100, 12329-12333, 1995.

Mikhailovskii, A. B., Aburjania, G. D., Lakhin, V. P., Mikhailovskaia, L. A., and Onishchenko, O. G.: On the Theory of Alfvén Waves, Plasma Phys. Contr. Fusion, 29, 1-25, 1987.

Mikhailovskaia, L. A.: Nonlinear dynamics of dipole drift vortices in plasma, Fizika Plasmi, 12, 879-881, 1986.

Mikhailovskii, A. B. and Pokhotelov, A. B.: Influence of the helicons and ion-cyclotron oscillations on Alfvén waves swinging in the magnetosphere plasma, Fizika Plasmi, 1, 1004-1012, 1975.

Mikhailovskii, A. B., Lakhin, V. P., Aburjania, G. D., Onishchenko, O. G., and Smolyakov, A. I.: On the alfvén vortices, Plasma Phys. Contr. Fusion, 29, 1-25, 1987.

Muller, W.-C., Biskamp, D., and Grappin, R.: Statistical anisotropy of magnetohydrodynamic turbulence, Phys. Rev. E., 67, 066302, doi:10.1103/PhysRevE.67.066302, 2003.

Narita, Y., Glassmeier, K.-H., Fränz, M., Nariyuki, Y., and Hada, T.: Observations of linear and nonlinear processes in the foreshock wave evolution, Nonlin. Processes Geophys., 14, 361-371, 2007, http://www.nonlin-processes-geophys.net/14/361/2007/.

Nezlin, M. V. and Snezhkin, E. N.: Rossby Vortices, Spiral Structures, Solitons: Springer-Verlag, Heidelberg, 1993.

Ng, C. S., Bhattacharjee, A., and Germaschewcki, K.: Anisotropic fluid turbulence in the interstellar medium and solar wind, Phys. Fluids, 10, 1954-1962, 2003.

Pecseli, H. L., Rasmussen, J. T., and Thomsen, K.: Nonlinear interaction of convective cells in plasmas, Phys. Rev. Lett, 52, 2148 2151, 1984

Petviashvili, V. I. and Yankov, V. V.: Solitons and Turbulence, in: Reviews of Plasma Physics, 14, edited by: Kadomtsev, B. B., Consultant Bureau, New York, 1987.

Petviashvili, V. I. and Pokhotelov, O. A.: Solitary Waves in Plasmas and in the Atmosphere: Gordon and Breach Science Publishers, Reading-Philadelphia, 1992.

Qian, J.: Nonequilibrium statistical mechanics of two-dimensional turbulence, Phy. Fluids, 27, 2412-2417, 1984.

Richardson, L. F.: Wheather Prediction of "Numerical Method", Cambr. Univ. Press, 1922.

Rosenbluth, M. N., Berk, H. L., and Doxas, I.: Effective diffusion in laminar convective, flows, Phys. Fluids, 30, 2636-2647, 1987.

Sagdeev, R. Z., Moiseev, S. S., Tur, A. V., and Yanovskii, V. V.: Problems of the theory of strong turbulence and topological solitons, in: Non-linear phenomena in plasma physics and hydrodynamics, edited by: Sagdeev, R. Z., Mir Publishers, Moscow, 131-182, 1986.

Semet, A., Mase, A., Peebles, W. A., Luhman, N. C., and Zweben, S.: Study of low-frequency microturbulence in the Microtor Tokamak by far-infrared laser scattering, Phys. Rev. Lett., 44, 1411-1414, 1980.
Sahraoui, F., Belmont, G., and Pincon, J. L.: Magnetic turbulent spectra in the magnetosheath: The k-filtering technique applied to Cluster II data, J. Geophys. Res., 108, A1335, doi:10.1029/2002JA009587, 2003.

Sahraoui, F., Belmont, G., Pinçon, J. L., Rezeau, L., Balogh, A., Robert, P., and Cornilleau-Wehrlin, N.: Magnetic turbulent spectra in the magnetosheath: new insights, Ann. Geophys., 22, 2283-2288, 2004, http://www.ann-geophys.net/22/2283/2004/.

Sahraoui, F., Belmont, G., Rezeau, L., and Cornilleau-Wehrlin, N.: Anisotropic turbulent spectra in the terrestrial magnetosheath as seen by the Cluster spacecraft, Phys. Rev. Lett., 96, 075002, doi:10.1103/Phys Rev Lett.96.075002, 2006.

Schekochihin, A. A., Cowley, S. C., and Dorland, W.: Interplanetary and interstellar turbulence, Plasma Phys. Contr. Fusion, 49, A195, doi:10.1088/0741-3335/49/5A/S16, 2007.

Shapiro, V. D. and Shevchenko, V. I.: Strong turbulence plasma oscillations, in: Basic Plasma Physics, 2, edited by: Galeev, A. A. and Sudan, R. N., North-Holand, Amsterdam, 1985.

Stasiewicz, K., Bellan, P., Chaston, C., Kletzing, C., Lysak, R., Maggs, J., Pokhotelov, O., Seyler, C., Shukla, P., Stenflo, L., Streltsov, A., and Wahlund, J.-E.: Small scale alfvénic structure in the aurora, Space Sci. Rev., 92, 423-533, 2000.

Sundkvist, D., Krasnoselskikh, V., Shukla, K., Vaivads, A., Andre, M., Buchert, S., and Reme, H.: In situ multi-satellite detection of coherent vortices as a manifestation of Alfvénic turbulence, Nature, 436, 825-828, doi:1038/nature04144, 2005.

Sutirin, G. G.: Long-lived planetary vortices and their evolution: Conservative intermediate geostrophic model, Chaos, 4, 203 212, 1994.

Tu, C. P. and Marsch, E.: MHD Structures, Waves and Turbulence in the Solar Wind: Observations and Theories, Kluwer Academic, Belgium. 1997.

Verkhoglyadova, O. P., Dasgupta, B., and Tsurutani, B. T.: Model for vortex turbulence with discontinuities in the solar wind, Nonlin. Processes Geophys., 10, 335-343, 2003, http://www.nonlin-processes-geophys.net/10/335/2003/.

Waltz, R. E.: Subcritical magnetohydrodynamic turbulence, Phys. Rev. Lett., 55, 1098-1101, 1985.

Weiland, J.: Nonlinear excitation of convection cells and anomalous diffusion in inhomogeneous plasmas, Phys. Rev. Lett., 44, 1411$1414,1977$.

Weissen, H., Hollenstein, Ch., and Benn, K.: Turbulent density fluctuations in the TCA Tokamak, Plasma Phys. Contr. Fusion, 30, 293-309, 1988.

Zimbardo, G.: Magnetic turbulence in space plasmas: in and around the Earths magnetosphere, Plasma Phys. Contr Fusion, 48, B295-B302, doi:10.1088/0741-3335/48/12B/S28, 2006. 